\title{
Manejo de citostáticos y salud reproductiva: revisión bibliográfica
}

\section{Cytostatic Agents and effects on reproductive health: a review of literature}

\author{
Maite Viñeta Ruiz ', Ma José Gallego Yanez', Ghino Patricio Villanueva ' \\ 1 Unidad Docente Madrid 1- Hospital Universitario Puerta de Hierro. Madrid. España. \\ Recibido: 18-07-13 \\ Aceptado: 22-10-13
}

\section{Correspondencia}

Maite Viñeta Ruiz

Hospital Universitario Puerta de Hierro. Madrid. España

Correo electrónico: maitevineta@hotmail.com

Resumen

Introducción: La utilización de agentes citostáticos ha mostrado suficiente evidencia científica en el origen de efectos carcinogénicos, mutagénicos y teratogénicos en seres humanos. El riesgo de exposición a estos agentes no se limita esencialmente a las personas que reciben dosis terapéuticas, también existe importante riesgo de exposición sobre la salud de los trabajadores que participan en la preparación, manipulación, administración y almacenamiento de estos medicamentos.

Objetivo general: Revisar la literatura científica para establecer la relación entre el manejo de citostáticos por el personal sanitario y los posibles efectos sobre la salud reproductiva.

Material y Métodos: Se realizó una revisión de la literatura existente a partir del año 2005 hasta el año 2012. La búsqueda se realizó por consulta directa y acceso por internet a la literatura recogida en las bases de datos de MEDLINE. Al introducir los filtros se obtuvo un total de 5 publicaciones de evidencia científica, las cuales fueron analizadas.

Resultados: En un meta-análisis de 2005 se encontraron datos de abortos espontáneos, fetos muertos, malformaciones congénitas, embarazos ectópicos y cáncer. En un estudio posterior de casos y control se encontraron datos de partos prematuros, bajo peso al nacer y dificultades para conseguir la concepción. En los estudios de cohortes se encontró evidencia de: en uno, de abortos espontáneos; en otro de malformaciones congénitas y cáncer y en otro se encontraron datos de bajo peso al nacer y anomalías congénitas, pero con menores cifras de lo esperado.

Discusión/Conclusiones: Los resultados encontrados no muestran una asociación estadística significativa, y por lo tanto sería de utilidad realizar estudios más potentes. A pesar de la existencia de Guías y Protocolos desde 2004, estudios posteriores a 2005 siguen evidenciando riesgos y efectos relacionados con la exposición, por lo tanto existe un margen para mejorar la evaluación del cumplimiento de dichos protocolos. A su vez existe rango de mejora en el campo de Vigilancia de la Salud.

Med Segur Trab (Internet) 2013; 59 (233) 426-443

Palabras clave: Citostáticos, salud reproductiva, efectos mutagénicos, carcinogénicos y teratogénicos. 


\section{Abstract}

Introduction: The use of cytostatic agents has shown enough scientific evidence in the origin of mutagenic and cancer effects, as well as teratogenicity in human beings. The risk of exposure to these agents is not only confined to patients receiving therapeutic doses, but also there is an important exposure risk on the health of workers participating in the preparation, handling, administration and storage of these agents.

General Objective: To review current literature in order to establish the relationship between cytostatic handling by health workers, and the possible effects on reproductive health.

Material and Methods: A literature review between 2005 to 2012 was carried out. The search was done by direct consultation and internet access to MEDLINE data. After screening, a total of 5 publications of scientific evidence were found and analyzed.

Results: In a meta-analysis in 2005, data on the following effects was found: spontaneous abortions, congenital deformities, ectopic pregnancies and cancer. Thereafter, in a case-control study, data on the following effects was found: premature childbirth, low weight at birth and difficulties in achieving conception. Cohort studies showed the following evidence: spontaneous abortions in one of them, congenital deformities and cancer in the second one, and low weight at birth and congenital deformities in the third one, but with lower figures than expected.

Discussion: The results of this study don't show a strong statistical association, and therefore more powerful studies would be recommended. Despite existing Guidelines from 2004, studies from 2005 still show risks and effects related to exposure; so there is a long way to go in improving adherence and evaluation of these Guidelines. Also there is place for improvement in the field of Health Surveillance.

$$
\text { Med Segur Trab (Internet) 2013; } 59 \text { (233) 426-443 }
$$

Key words: Cytostatic agents, reproductive health, mutagenicity, teratogenicity and cancer effects. 


\section{INTRODUCCIÓN}

La palabra citostático proviene del griego kytos (célula) y stasis (detención). Su definición pregona: sustancia que detiene la multiplicación de las células. Los compuestos citostáticos se encuentran clasificados según la International Agency for Research on Cancer $(\text { IARC })^{1}$.

Los agentes citostáticos o fármacos antineoplásicos se utilizan cada vez más en el tratamiento de muchas enfermedades y en especial de aquellas relacionadas con el cáncer, estos fármacos no sólo afectan a las células tumorales sino también a todas las células del organismo. Los datos de la Agencia Internacional para la Investigación del Cáncer y diversos estudios sobre su tratamiento, han mostrado suficiente evidencia científica para determinar los efectos carcinogénicos, mutagénicos y teratogénicos de muchos de estos agentes citostáticos en seres humanos ${ }^{1-3}$.

Dos estudios de revisión sistemática sobre el tratamiento de la mujer embarazada con cáncer y el uso de citotóxicos, agentes dirigidos e inmunoterapia, muestran una clara relación en la producción de tumores sólidos y hematológicos ${ }^{4,5}$. Asimismo, hay evidencia de que los citostáticos al administrarse en dosis terapéuticas a pacientes oncológicas embarazadas, causan malformaciones congénitas y muerte fetal ${ }^{6-8}$. Aunque se pensaba que el riesgo de exposición a estos agentes citostáticos se limitaba esencialmente a las personas que recibían dosis terapéuticas, se ha demostrado en estudios recientes que existe importante riesgo de exposición sobre la salud de los trabajadores que participan en la preparación, manipulación, administración y almacenamiento de estos medicamentos. Otra forma de exposición profesional a agentes citostáticos es el contacto con los pacientes en tratamiento, así como la manipulación de las excretas y ropas ${ }^{9,10}$; por lo tanto el rango de trabajadores en riesgo se incrementa a personal auxiliar, de limpieza y de laboratorio.

Aunque la intensidad de la exposición es menor en comparación a los pacientes que reciben tratamiento, las exposiciones ocupacionales suelen ser acumulativas en el tiempo. A pesar de la introducción de directrices de seguridad y medidas de protección ${ }^{11}$, el personal sanitario puede estar todavía expuesto a los agentes citostáticos, como así lo demuestran los niveles detectables de biomarcadores en orina, los daños del ADN y las aberraciones cromosómicas observadas en los linfocitos y las células exfoliadas bucales ${ }^{12-15}$.

Varios estudios llevados a cabo en hospitales han demostrado niveles detectables de agentes citostáticos en el aire ${ }^{16,17}$, en las superficies ${ }^{18-21}$, en los guantes ${ }^{22,23}$ y en diferentes partes del cuerpo ${ }^{22,24,25}$. Diferentes métodos de control biológico se han desarrollado para detectar la exposición ocupacional a agentes citostáticos ${ }^{26}$ y se ha estudiado ampliamente la presencia de estos fármacos en la orina del personal sanitario ${ }^{24,27-29}$. Asimismo, el Instituto Nacional de Seguridad e Higiene en el Trabajo en sus notas técnicas de prevención sobre la exposición a citostáticos, advierte de niveles detectables de éstos en el ámbito sanitario, pues realiza una clasificación en función del tipo de citostático y sus niveles encontrados en superficies, guantes, viales y orina ${ }^{30}$ (Anexos 1, 2, 3, 4).

Algunos estudios han sugerido que los agentes citostáticos a niveles de exposición ocupacional pueden ser tóxicos para la reproducción, aunque estos no son concluyentes ni consistentes ${ }^{31-36}$. Otros estudios han demostrado que las enfermeras expuestas a citostáticos presentan dificultades para concebir y que los hijos de éstas, expuestas a citostáticos durante el embarazo, corren mayor riesgo prenatal para el desarrollo de anomalías congénitas, riesgo de parto prematuro y bajo peso al nacer, aunque no mayor riesgo de aborto espontáneo y muerte fetal ${ }^{37}$.

Estudios anteriores relacionan el riesgo aumentado de infertilidad en hombres ${ }^{38}$ y el de aborto en esposas de trabajadores expuestos frente a las no expuestas ${ }^{34}$; a pesar de encontrar cierta relación, ambos estudios no llegan a ser estadísticamente significativos.

En el año 2005, George Dranitsaris et al. publican un estudio sobre una revisión sistemática de la bibliografía y realizan un meta-análisis, encontrando asociación entre la exposición a fármacos antineoplásicos y abortos espontáneos; sin embargo no se encontró 
asociación estadísticamente significativa entre exposición con malformaciones congénitas y muerte fetal ${ }^{13}$.

Por todo lo anteriormente expuesto, se ha realizado una revisión de la literatura existente a partir del 2005 hasta 2012, para determinar nueva evidencia entre la exposición ocupacional a agentes citostáticos y los riesgos sobre la salud reproductiva.

\section{OBJETIVOS}

\section{Objetivo general}

Revisar la literatura científica para establecer la relación entre el manejo de citostáticos por el personal sanitario y los posibles efectos sobre la salud reproductiva inducidos por mecanismos genotóxicos y teratógenos.

\section{Objetivos específicos}

1. Determinar los mecanismos y factores que influyen en la exposición a citostáticos.

2. Categorizar los efectos resultantes de la exposición a citostáticos.

3. Detallar los mecanismos de vigilancia y control de la exposición.

\section{Material y métodos}

Se realizó una revisión bibliográfica sistemática de la literatura.

Para identificar las fuentes de información se realizó la búsqueda por consulta directa y acceso por internet a la literatura recogida en las bases de datos de scielo, osh y MEDLINE (Medical Literature Analysis and Retrieval System Online) vía PubMed, encontrando mejores resultados en MEDLINE; por lo que se estableció un criterio de búsqueda utilizando para MEDLINE los términos del Medical Subject Headings (MeSH).

Se consideró adecuado el uso de los descriptores «Occupational Health”, "Occupational Exposure», "Antineoplastic Agents", "Cytostatic Agents", "Pharmaceutical Preparations", "Pregnancy", "Pregnancy Outcome». Se aplicaron los limites "Humans", y se utilizaron los conectores booleanos "OR" y "AND".

Estrategia de búsqueda en MEDLINE

((“Occupational Health”[Mesh]) OR "Occupational Exposure"[Mesh]) AND ((“Antineoplastic Agents”[Mesh]) OR ("Cytostatic Agents"[Mesh]) OR "Pharmaceutical Preparations"[Mesh]) AND (("Pregnancy”[Mesh]) OR

"Pregnancy Outcome"[Mesh])

Se incluyó la documentación según los siguientes criterios de búsqueda:

- Los documentos deberían mostrar una asociación entre agentes citostáticos y riesgo para la salud reproductiva en personal sanitario.

- La población de estudio debería incluir: enfermeras(os), médicos, farmacéuticos, auxiliares de enfermería, laboratorio y limpieza.

- Año de publicación: desde 2005 hasta 2012 (última actualización el 31 de diciembre). Se escogió como inicio de la búsqueda el año 2005 porque en aquel año se publicó una revisión sistemática y meta-análisis sobre exposición a agentes citostáticos y riesgo en salud reproductiva ${ }^{13}$.

- Se incluyeron para este estudio los siguientes documentos: meta-análisis, ensayo clínico, estudios experimentales, casos y controles, cohortes, estudios observacionales y opinión de expertos.

- Además se realizó búsqueda manual de literatura de aquellos documentos identificados en la bibliografía de la literatura seleccionada por el buscador. 
- Se seleccionó la documentación a texto completo que se consideró pertinente.

- Se realizó la búsqueda de los documentos publicados en inglés o español.

Se excluyó documentación encontrada como publicación duplicada en diferentes bases de datos.

Con la finalidad de obtener literatura concerniente a personal sanitario de sexo masculino se cruzaron los descriptores relacionados a citostáticos y exposición ocupacional, anteriormente utilizados, con los siguientes descriptores: "Infertility", "Infertility, Male", “Azoospermia”[Mesh] OR "Oligospermia”[Mesh], sin la obtención de documentación adicional.

Cada uno de los artículos se evaluó de manera independiente por los tres autores de la presente revisión y se seleccionaron de manera consensuada aquellos que se incluirían en el estudio.

Se presenta una tabla comparativa (Figura 1) con datos relevantes y con la finalidad de facilitar la comprensión de los resultados de los documentos revisados. Además se les asignó un nivel de evidencia científica de acuerdo a los criterios del Scottish Intercollegiate Guidelines Network (SIGN).

\section{Resultados de la búsqueda}

Se localizaron un total de 37 publicaciones entre 1974 y 2012. Al introducir los filtros anteriormente descritos se obtuvo un total de 10 publicaciones y tras aplicar los criterios de inclusión y exclusión, se seleccionaron un total de 4 publicaciones para la presente revisión. Se obtuvo un meta-análisis, un estudio de casos y controles, un estudio de cohortes y una carta al editor.

Se realizó también la búsqueda secundaria de la bibliografía mediante PubMed y se encontró literatura que cumplía con los criterios de inclusión y exclusión planteados. Se obtuvieron 2 estudios de cohortes y una opinión de expertos.

En total se seleccionaron 7 publicaciones referentes a exposición a citostáticos y salud reproductiva desde el año 2005 hasta 2012 de los cuales 5 tenían adecuada evidencia científica (Figura 1).

\section{Figura 1}

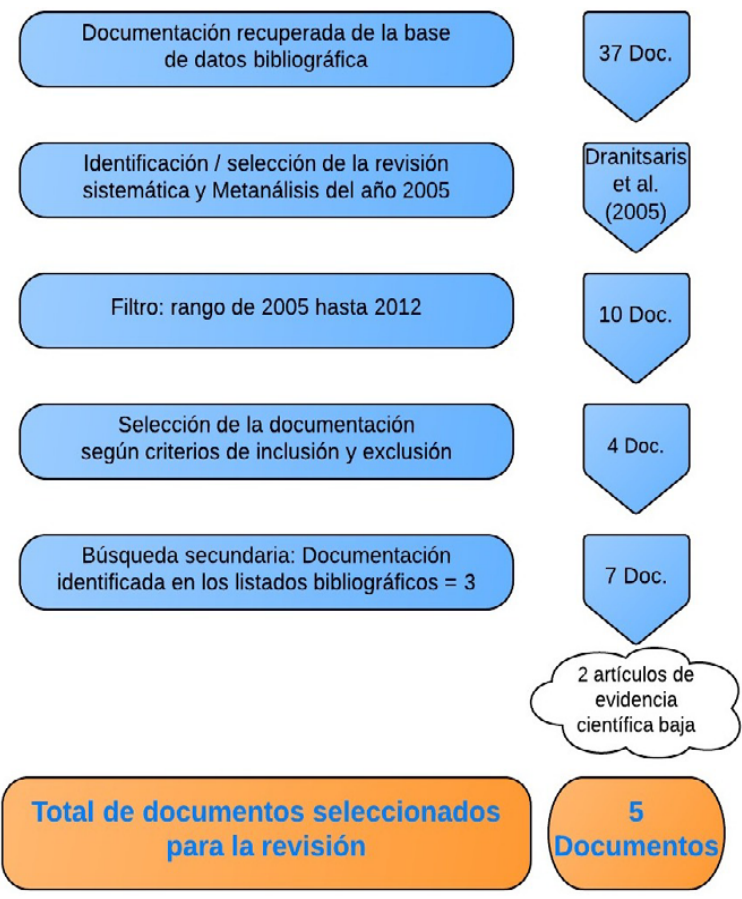


Tabla 1. Tabla Resumen de los artículos seleccionados (en negrita los 5 de mayor evidencia científica según SIGN)

\begin{tabular}{|c|c|c|c|c|c|c|}
\hline $\begin{array}{c}\text { Autor / Año } \\
\text { de publicación }\end{array}$ & Diseño & Población & $\begin{array}{c}\text { Variable } \\
\text { de Exposición }\end{array}$ & Variable resultado & Resultados & $\begin{array}{l}\text { Nivel de } \\
\text { evidencia }\end{array}$ \\
\hline $\begin{array}{l}\text { George } \\
\text { Dranitsaris } \\
\text { et al. } 2005^{13} \text {. }\end{array}$ & $\begin{array}{l}\text { - Meta-análisis. } \\
\text { - Revisión } \\
\text { sistemática. }\end{array}$ & $\begin{array}{l}14 \text { Publicaciones } \\
\text { de casos y } \\
\text { controles, } \\
\text { cohortes } \\
\text { y estudios } \\
\text { transversales. }\end{array}$ & $\begin{array}{l}\text { Agentes } \\
\text { citostáticos. }\end{array}$ & $\begin{array}{l}\text { - Abortos } \\
\text { espontáneos. }\end{array}$ & $\begin{array}{l}\text { - Se encontró asociación } \\
\text { entre la exposición a } \\
\text { fármacos antineoplásicos } \\
\text { y abortos espontáneos } \\
\text { (OR=1.46; 95\%; } \\
\text { IC= 1.11-1.92). } \\
\text { - Sin embargo no se } \\
\text { encontró asociación } \\
\text { estadísticamente significativa } \\
\text { entre exposición con } \\
\text { malformaciones congénitas } \\
\text { (OR=1.64; 95\%; IC=0.91-2.94) } \\
\text { y muerte fetal (OR=1.16; 95\%; } \\
\text { IC=0.73-1.82). }\end{array}$ & $1-$ \\
\hline $\begin{array}{l}\text { Wouter } \\
\text { Fransman et } \\
\text { al. } 2007^{25} \text {. }\end{array}$ & $\begin{array}{l}\text { Casos y } \\
\text { controles. }\end{array}$ & $\begin{array}{l}\mathrm{N}=4393 \\
\text { (Enfermería) }\end{array}$ & $\begin{array}{l}\text { Agentes citostáticos } \\
\text { (Ciclofosfamida) }\end{array}$ & $\begin{array}{l}\text { - Parto } \\
\text { prematuro. } \\
\text { - Bajo peso al } \\
\text { nacer. } \\
\text { - Aumento del } \\
\text { tiempo para } \\
\text { concebir. }\end{array}$ & $\begin{array}{l}\text { - Las enfermeras altamente } \\
\text { expuestas a fármacos } \\
\text { antineoplásicos tardaban } \\
\text { en conseguir la concepción } \\
\text { (Razón de riesgo ajustado = } \\
0.8 ; \text { IC = 0.6-0.9). } \\
\text { - La exposición a fármacos } \\
\text { antineoplásicos se asocia } \\
\text { a parto prematuro (OR } \\
\text { incremento por unidad } \\
\text { Ln[exposición]= 1.08; } \\
\text { IC = 1.00-1.17) y bajo peso } \\
\text { al nacer (OR incremento } \\
\text { por unidad Ln[exposición]= } \\
1.11 ; \text { IC = 1.01-1.21). }\end{array}$ & $2++$ \\
\hline $\begin{array}{l}\text { Laura T } \\
\text { Arbour et al. } \\
2010^{47} \text {. }\end{array}$ & Cohorte. & $\begin{array}{l}\mathrm{N}=23222 \\
\text { nacimientos } \\
\text { (Enfermería) }\end{array}$ & $\begin{array}{l}\text { Gases anestésicos, } \\
\text { antineoplásicos, } \\
\text { radiaciones } \\
\text { ionizantes, } \\
\text { esterilizantes, } \\
\text { desinfectantes } \\
\text { y agentes } \\
\text { infecciosos. }\end{array}$ & $\begin{array}{l}\text { - Anomalías } \\
\text { congénitas. } \\
\text { - Bajo peso al } \\
\text { nacer. }\end{array}$ & $\begin{array}{l}\text { - Reportaron que los hijos } \\
\text { de las enfermeras expuestas } \\
\text { tienen menor número } \\
\text { de anomalías congénitas } \\
(\mathrm{OR}=0.84 ; 95 \% \mathrm{IC}, 0.78-0.90) \\
\text { y menor número de recién } \\
\text { nacidos con bajo peso al } \\
\text { nacer (OR=0.90;95\%,0.83- } \\
0.98) \text { que el personal no } \\
\text { expuesto. }\end{array}$ & $2++$ \\
\hline $\begin{array}{l}\text { Pamela A } \\
\text { Ratner et al. } \\
2010^{50} \text {. }\end{array}$ & Cohorte. & $\begin{array}{l}\mathrm{N}=56213 \\
\text { (Enfermería) }\end{array}$ & $\begin{array}{l}\text { Agentes } \\
\text { citostáticos. }\end{array}$ & $\begin{array}{l}\text { - Anomalías } \\
\text { congénitas. }\end{array}$ & $\begin{array}{l}\text { - Los hijos de las } \\
\text { enfermeras que trabajaban } \\
\text { en un centro de cáncer o en } \\
\text { una unidad de enfermería } \\
\text { oncológica presentaban } \\
\text { riesgo de anomalías } \\
\text { congénitas oculares (OR= } \\
\text { 3.46, 95\% IC = 1.08-11.14). } \\
\text { También existe riesgo de } \\
\text { otras anomalías congénitas. } \\
\text { - Las enfermeras } \\
\text { ocupacionalmente } \\
\text { expuestas a fármacos } \\
\text { antineoplásicos tuvieron un } \\
\text { riesgo elevado de cáncer de } \\
\text { mama y recto. }\end{array}$ & $2+$ \\
\hline
\end{tabular}




\begin{tabular}{|c|c|c|c|c|c|c|}
\hline $\begin{array}{c}\text { Autor / Año } \\
\text { de publicación }\end{array}$ & Diseño & Población & $\begin{array}{c}\text { Variable } \\
\text { de Exposición }\end{array}$ & Variable resultado & Resultados & $\begin{array}{l}\text { Nivel de } \\
\text { evidencia }\end{array}$ \\
\hline $\begin{array}{l}\text { Cristina C } \\
\text { Lawson et } \\
\text { al. } 2012^{49} \text {. }\end{array}$ & Cohorte. & $\begin{array}{l}\mathrm{N}=8461 \\
\text { (Enfermería) }\end{array}$ & $\begin{array}{l}\text { Agentes } \\
\text { citostáticos, } \\
\text { agentes } \\
\text { esterilizantes, } \\
\text { gases anestésicos, } \\
\text { antivirales y } \\
\text { radiación X. }\end{array}$ & $\begin{array}{l}\text { - Aborto } \\
\text { espontáneo. }\end{array}$ & $\begin{array}{l}\text { - Reportaron que la } \\
\text { exposición a fármacos } \\
\text { antineoplásicos se } \\
\text { asociaron a un mayor riesgo } \\
\text { de aborto espontáneo. (OR, } \\
2.13 ; 95 \% \text { IC, 1.39-3.27). }\end{array}$ & $2++$ \\
\hline $\begin{array}{l}\text { Robin } \mathbf{M} \\
\text { Lally. } 2007^{48} \text {. }\end{array}$ & $\begin{array}{l}\text { Opinión de } \\
\text { expertos. }\end{array}$ & & $\begin{array}{l}\text { Agentes } \\
\text { citostáticos. }\end{array}$ & & $\begin{array}{l}\text { - Se hace hincapié en } \\
\text { la importancia de evitar } \\
\text { la exposición excesiva a } \\
\text { la quimioterapia cuando } \\
\text { las enfermeras (os) o sus } \\
\text { parejas están embarazadas } \\
\text { o planifican concebir. } \\
\text { Dado que las vías más } \\
\text { probables de exposición } \\
\text { para las enfermeras y } \\
\text { los farmacéuticos son la } \\
\text { inhalación y absorción por } \\
\text { la piel (NIOSH, 2004); el uso } \\
\text { de equipos de protección } \\
\text { individual apropiado y la } \\
\text { adhesión a las directrices de } \\
\text { prevención son los métodos } \\
\text { principales de la reducción } \\
\text { de riesgos. }\end{array}$ & \\
\hline $\begin{array}{l}\text { Wilson } \\
\text { Cañon } \\
\text { Montañez et } \\
\text { al. } 2010^{46} \text {. }\end{array}$ & $\begin{array}{l}\text { Revisión } \\
\text { narrativa de la } \\
\text { literatura. }\end{array}$ & & $\begin{array}{l}\text { Drogas } \\
\text { antineoplásicas. }\end{array}$ & & $\begin{array}{l}\text { - Hay evidencia clara de } \\
\text { asociación e incremento del } \\
\text { riesgo para el desarrollo de } \\
\text { desenlaces reproductivos } \\
\text { negativos en el personal } \\
\text { con exposición ocupacional } \\
\text { a fármacos antineoplásicos. }\end{array}$ & 4 \\
\hline
\end{tabular}

\section{RESULTADOS}

A continuación se describen los resultados observados en la revisión realizada por los autores que llevaron a cabo el meta-análisis, y posteriormente se presentan los principales resultados de los trabajos revisados.

\section{Meta-análisis}

George Dranitsaris et al ${ }^{(13)}$ en 2005 realizan un meta-análisis y una revisión sistemática para evaluar la hipótesis de que los profesionales de la salud que trabajan con drogas antineoplásicas (enfermeras, farmacéuticos y auxiliares de farmacia) tienen:

1. Un riesgo aumentado de cáncer (hematológico sobre todo).

2. Complicaciones reproductivas (abortos espontáneos, muerte fetal y malformaciones congénitas).

3. Eventos tóxicos agudos (perdida de pelo, náuseas, etc.).

Analizaron 14 estudios epidemiológicos en humanos llevados a cabo desde 1996 a diciembre de 2004, siete de los cuales fueron adecuados, de los cuales cinco son series de casos, uno de cohortes y el resto transversales. 
No hubo una asociación estadísticamente significativa entre la exposición a fármacos citotóxicos y malformaciones congénitas (OR=1.64,IC 95\%:0.91 a 2,94) o muerte fetal (OR=1.16,95\% IC:1.11-1.92).

Los resultados de esta revisión sistemática identificaron una asociación entre exposición a quimioterápicos y abortos espontáneos (OR=1.46, IC 95\% :1.11-1.92).

Tabla 2. Principales resultados del meta-análisis

\begin{tabular}{|c|c|c|c|}
\hline EFECTOS & RESULTADOS & AUTOR & $\begin{array}{c}\text { TIPO } \\
\text { DE ESTUDIO }\end{array}$ \\
\hline $\begin{array}{l}\text { Malformaciones } \\
\text { congénitas }\end{array}$ & $\begin{array}{l}\text { Seis estudios registraron datos sobre } \\
\text { malformaciones congénitas pero no hubo una } \\
\text { asociación significativa entre la exposición } \\
\text { a fármacos citotóxicos y malformaciones } \\
\text { congénitas. }\end{array}$ & $\begin{array}{l}\text { Hemmminiki et } \\
\text { al. }{ }^{35} \\
\text { Mc Donald et al. }{ }^{39} \\
\text { Mc Abee et al. }{ }^{41} \\
\text { Peelen et al. }{ }^{44} \\
\text { Lorente et al. }{ }^{45}\end{array}$ & $\begin{array}{l}\text { Casos y control } \\
\text { Estudio Transversal } \\
\text { Estudio Transversal } \\
\text { Estudio Transversal } \\
\text { Casos y control }\end{array}$ \\
\hline $\begin{array}{l}\text { Abortos } \\
\text { espontáneos }\end{array}$ & $\begin{array}{l}\text { Se registraron siete estudios. Solamente } \\
\text { el estudio de Selevan et al., que focalizó } \\
\text { la exposición a fármacos citotóxicos en } \\
\text { enfermeras que trabajaban en departamentos } \\
\text { de administración de quimioterapia, y } \\
\text { su relación con los efectos en la salud } \\
\text { reproductiva fue incluido en el meta-análisis, } \\
\text { encontrando que existe un pequeño riesgo } \\
\text { incrementado entre este personal y los } \\
\text { abortos espontáneos. Se excluyó el artículo } \\
\text { de Hemminiki y el de McAbee. El estudio de } \\
\text { Valanis incluía a farmacéuticos y asistentes de } \\
\text { farmacia. }\end{array}$ & $\begin{array}{l}\text { Hemmminiki et al. }{ }^{35} \\
\text { Selevan et al. }{ }^{31} \\
\text { Valanis et al. }{ }^{34} \\
\text { Stucker et al. }{ }^{36} \\
\text { Skov et al. }{ }^{36} \\
\text { Mc Abee et al. }{ }^{41} \\
\text { Peelen et al. }{ }^{44}\end{array}$ & $\begin{array}{l}\text { Casos y control } \\
\text { Casos y control } \\
\text { Estudio Transversal } \\
\text { Estudio Transversal } \\
\text { Estudio Cohorte } \\
\text { Estudio Transversal } \\
\text { Estudio Transversal }\end{array}$ \\
\hline Muerte fetal & $\begin{array}{l}\text { Solamente tres estudios evidenciaron fetos } \\
\text { muertos pero no se detectó asociación } \\
\text { significativa. }\end{array}$ & $\begin{array}{l}\text { Valanis et al. }{ }^{34} \\
\text { Mc Abee et al. }{ }^{41} \\
\text { Peelen et al. }{ }^{44}\end{array}$ & $\begin{array}{l}\text { Estudio Transversal } \\
\text { Estudio Transversal } \\
\text { Estudio Transversal }\end{array}$ \\
\hline $\begin{array}{l}\text { Embarazo } \\
\text { ectópico }\end{array}$ & Tres estudios reportaron embarazos ectópicos. & $\begin{array}{l}\text { Skov et al. }{ }^{36} \\
\text { Saurel et al. }{ }^{43} \\
\text { Bouyer et al. }{ }^{43}\end{array}$ & $\begin{array}{l}\text { Estudio Cohorte } \\
\text { Estudio Transversal } \\
\text { Casos y control }\end{array}$ \\
\hline Efectos tóxicos & $\begin{array}{l}\text { Solamente dos estudios evaluaron esto, pero } \\
\text { no se llegó a conclusiones por un inadecuado } \\
\text { registro de los casos. }\end{array}$ & Valanis et al. ${ }^{38}$ & Estudio Transversal \\
\hline Cáncer & $\begin{array}{l}\text { Se registraron catorce cánceres, incluidos } \\
\text { tres hematológicos, entre enfermeras que } \\
\text { preparaban y administraban citotóxicos, } \\
\text { pero la falta de un grupo control sin datos } \\
\text { suficientes impidió la interpretación, según el } \\
\text { meta-análisis. } \\
\text { Mama: se detectaron siete casos entre } \\
\text { enfermeras especializadas en cuidados } \\
\text { oncológicos, pero el número de expuestas a } \\
\text { drogas citotóxicas no estaba claro, haciendo } \\
\text { difícil la asociación con el riesgo. }\end{array}$ & $\begin{array}{l}\text { Skov et al. }{ }^{36} \\
\text { Gunnarsdottir } \\
\text { et al. }{ }^{42}\end{array}$ & $\begin{array}{l}\text { Estudio cohorte } \\
\text { retrospectivo } \\
\text { Casos y control }\end{array}$ \\
\hline
\end{tabular}

\section{Estudios de Casos y Controles}

Wouter Fransman et al. ${ }^{37}$ en 2007, estudiaron la asociación entre la exposición cutánea a fármacos antineoplásicos en las enfermeras y los efectos sobre la reproducción. Se utilizó la ciclofosfamida como marcador de exposición.

Se realizó el estudio en 121 hospitales de los Países Bajos. Se incluyeron 4.393 enfermeras en edades reproductivas expuestas y no expuestas, a las que se les realizó un cuestionario entre 1990 y 1997, mientras trabajaban por lo menos durante dos meses en 
un servicio de Oncología y otros servicios preseleccionados. Se formularon preguntas acerca de los resultados del embarazo, los riesgos relacionados con el trabajo y estilo de vida. Había 1.519 enfermeras que cumplían con los requisitos para el estudio.

Las enfermeras pertenecientes a los grupos expuestos tenían una edad similar en el momento el embarazo, con un estilo de vida similar y similares niveles de forma física.

Las enfermeras altamente expuestas a fármacos antineoplásicos tardaban en conseguir la concepción (Razón de riesgo ajustada de 0,8; IC del 95\%, IC=0,6 a 0,9). La proporción de desórdenes en la reproducción sugería un incremento de niños con bajo peso al nacer más alto en enfermeras expuestas $(\mathrm{OR}=2,1 ; \mathrm{IC}=0,9-4,7)$. Las enfermeras con baja exposición también llevaban apareado un incremento del riesgo de niños con bajo peso.

La exposición a fármacos antineoplásicos se asocia a parto prematuro [OR incremento por unidad Ln (exposición) $=1.08$, IC $=1.00-1.17$ ] y bajo peso al nacer [OR incremento por unidad en Ln (exposición)=1,11, IC 1.01-1,21].

El aborto espontáneo, la muerte fetal, las anomalías congénitas, y el sexo de la descendencia no parecían estar relacionado con exposición a los fármacos antineoplásicos.

\section{Estudios de cohortes}

Pamela A Ratner et al. ${ }^{50} 2010$, estudiaron la incidencia de cáncer y los resultados adversos en el embarazo en las enfermeras.

Se realizó un estudio de cohortes en una población de enfermeras $(\mathrm{N}=56.213)$ que trabajaban en una unidad de Oncología o en un centro de tratamiento del cáncer en Canadá entre el año 1974 y 2000. La exposición fue evaluada por dos métodos: Primero calculando el número de años que habían trabajado las enfermeras en un centro Oncológico o unidades de Oncología; segundo mediante la estimación de la exposición ponderada (unos 15 días) a partir de una encuesta telefónica realizada a farmacéuticos y enfermeras sobre la frecuencia de uso de fármacos antineoplásicos. Los riesgos relativos se calcularon mediante la regresión de Poissón para el cáncer y la incidencia y la OR se calculó para las anomalías congénitas, muerte fetal, bajo peso al nacer y la incidencia de partos prematuros con IC 95\%.

El estudio realizado con el primer método reveló que las enfermeras que habían trabajado en un centro oncológico o unidad de enfermería oncológica tenían un mayor riesgo de cáncer de mama $(\mathrm{RR}=1.83$, IC 95\%=1.03-3,23) y sus hijos estaban en riesgo de anomalías congénitas oculares (OR=3,46, IC 95\%=1,08-11,14). Los riesgos para todos los cánceres excepto el de piel, pero sí el melanoma $(\mathrm{RR}=1,28,95 \%$ IC = 0,83 a 1,79, 21 casos), o específicamente para los cánceres de útero $(\mathrm{RR}=2,58,95 \% \mathrm{CI}=0,96$ a 6,94) fueron elevados, pero no fueron estadísticamente significativos. Hubo casos de leucemia observados entre aquellos que han trabajado en Oncología o un centro Oncológico.

Con el segundo método el riesgo de cáncer rectal fue significativamente elevado ( $R R=1,87,95 \% \mathrm{CI}=1,07$ a 3,29), y el riesgo de cáncer de mama fue elevado, pero no fue estadísticamente significativo $(R R=1,12,95 \%$ IC $=0,89$ a 1,39$)$.

Entre los hijos de madres que habían trabajado alguna vez en las unidades de enfermería de Oncología o un centro de cáncer durante su embarazo (primer método), el riesgo de anomalías o malformaciones congénitas del sistema circulatorio o sistema musculo-esquelético fue elevado, aunque no estadísticamente significativo. Sin embargo, el riesgo de anomalías oculares fue estadísticamente significativo 3,46 (IC 95\% = 1,08- 11,14), pero basado en sólo 3 casos.

No hubo diferencias estadísticamente significativas ni aumento del riesgo de anomalías congénitas en los hijos de madres con la exposición a los fármacos antineoplásicos durante el primer trimestre del embarazo o durante los 10 años anteriores al embarazo (segundo 
método). Sin embargo, el riesgo de paladar hendido o labio, para el período de exposición de 10 años, fue notablemente alto, de 1,84 (IC 95\% = 0,75-4,49).

No había riesgo de incremento estadísticamente significativo de leucemia, otros tipos de cáncer, muerte fetal, bajo peso al nacer o prematuridad.

A pesar de que la muestra tenía un gran tamaño, los resultados de este estudio vienen limitados porque tienen un escaso poder estadístico, asociado a escasez de resultados, a la baja prevalencia de la exposición y pérdida de asociaciones. De modo que una asociación estadísticamente significativa puede haber ocurrido por pura casualidad y además como es común en los estudios de cohortes históricas no se dispone de información acerca de los posibles factores de confusión. Otras investigaciones, incluyendo mediciones más detalladas de las exposiciones y dosis recibidas, pueden ser útiles en la evaluación de los mecanismos por los que la exposición ocupacional a los fármacos antineoplásicos pueda actuar como factor de riesgo para el cáncer, y causar efectos adversos en la reproducción.

Christina C Lawson et al. ${ }^{49}$ en 2012, estudiaron la relación entre la exposición ocupacional entre las enfermeras y el riesgo de aborto espontáneo en 1989 entre enfermeras de edades comprendidas entre 25 y 42 años en 14 estados de Estados Unidos.

Se realizó un estudio de cohortes prospectivo entre 7.482 participantes elegidos de entre 8.461 participantes, 6.707 embarazos terminaron en nacidos vivos y 775 (10\%) en abortos espontáneos. El 74\% de estos terminó antes de las 12 semanas de embarazo $(n=575)$ El año de los embarazos osciló entre 1993-2002 (el principal año fue 1996), con el 82\% de ellas entre 1993 y 1998. La tasa de aborto espontáneo varía según la zona donde se trabaja, las tasas más bajas fueron para médicos de áreas quirúrgicas y críticos (8,4\% y 8,8\%, respectivamente), y las tasas más altas en Oncología (13,1\%) El 32\% de las enfermeras especificó otra área (11,0\% tasa de aborto espontáneo). Se tuvieron en cuenta variables como edad, tiempo trabajado, turnicidad y tiempo de exposición.

Las mujeres nulíparas tenían más riesgo de aborto espontáneo, también había tasas más altas de abortos entre las consumidoras de bebidas con cafeína y el alcohol y fumar cigarrillos también era más común entre los embarazos que terminan en aborto espontáneo.

Los resultados demostraron que las exposiciones repetidas a los fármacos antineoplásicos se asociaron a un mayor riesgo de aborto espontáneo (OR=2,13, IC 95\%, IC=1,39-3,27) de casi el doble que en el personal no expuesto.

Laura T Arbour et al. ${ }^{47}$ en 2010, realizaron un estudio de cohorte retrospectiva entre las enfermeras en British Columbia (BC). En 2003, se obtuvieron datos sobre 56.176 recién nacidos que se registraron entre 1974 y el año 2000. El estudio fue aprobado por la Universidad de British Columbia por el Comité de Ética de Investigación. Después de la verificación de los datos y el examen de los archivos, los resultados se obtuvieron sobre 23.222 nacimientos. Múltiples resultados reproductivos adversos fueron tratados como resultados independientes.

Una variedad de productos químicos inherentes a los entornos profesionales tienen el potencial de influir en el desarrollo prenatal, lo que resulta en efectos adversos en el nacimiento como anomalías congénitas, mortinatos, bajo peso al nacer, y prematuridad. Aproximadamente el $\mathbf{1 5 - 2 5 \%}$ de las anomalías congénitas se deben a enfermedades genéticas conocidas (por ejemplo, anomalías cromosómicas) y los casos restantes se sospecha que tienen algún grado de influencia ambiental.

Los trabajadores de salud están expuestos a una variedad de riesgos laborales que puede resultar en efectos adversos en la reproducción. Hay más de 2,5 millones de enfermeras registradas que trabajan en los Estados Unidos y 250.000 en Canadá, que están potencialmente expuestas a los gases anestésicos, medicamentos antineoplásicos, radiaciones ionizantes, agentes esterilizantes, desinfectantes y agentes infecciosos que pueden sufrir alguna alteración en la reproducción. 
Existen numerosos informes de casos que describen riesgos para la salud en enfermería, pero la evidencia de riesgos reproductivos relacionados con la exposición laboral para las enfermeras es relativamente escasa. Un registro basado en estudio de cohorte de las enfermeras en Noruega durante 1970-1973 mostró que los niños primogénitos de madres que eran enfermeras tuvieron un riesgo relativo elevado de malformaciones en comparación con madres que no eran enfermeras. Un estudio terminado en Atlanta, Georgia que compara los casos de defectos de nacimiento en función de áreas geográficas y de la raza en los controles sanos mostró que la descendencia de las madres que trabajan en el campo de la enfermería tuvo un riesgo mayor de defectos en el parto. Por el contrario en otros dos estudios se observó una menor proporción de defectos en el nacimiento en los hijos de las enfermeras en comparación con el resto de la población.

Los objetivos de este estudio fueron determinar:

a) si una cohorte de mujeres enfermeras en la provincia de British Columbia (BC), en comparación con la población general del BC, tenía una elevada prevalencia de anomalías congénitas, mortinatos, bajo peso al nacer y prematuridad en su descendencia

b) si ciertas características maternas e infantiles se asociaron con estos resultados.

Se tuvieron en cuenta las siguientes variables: edad gestacional, peso al nacimiento, año de nacimiento, edad de la madre en el alumbramiento y nivel de instrucción de la madre.

Los casos de anomalías congénitas (al menos una) fue significativamente inferior (1.567 observadas versus 1.846 esperadas; OR 0.84, 95\% IC=0.78-0.90) a la población general al igual que los casos del bajo peso al nacer (1.138 observado frente al 1.260 esperado; OR 0.90; IC=0.83-0.98), sin embargo el alto peso al nacer y los embarazos múltiples fueron significativamente mayores. También las anomalías cromosómicas fueron menores de lo esperado. Las reducciones más notables fueron en los defectos del corazón (SIR 0,52, IC 95\% 0.45-0.61), labio leporino y paladar (SIR 0,47, IC 95\% 0,34-0,65) y el número de casos con anomalías múltiples (SIR 0,43, IC 95\% 0.26-0.67).

Hubo más casos de anomalías congénitas en los hombres (7,4\% vs 6,1\%; OR 1,23, 95\% IC=1,11-1,36), atribuible a un aumento de la prevalencia de las verrugas genitales. Las enfermeras del hospital dieron a luz con mayor edad en comparación con el resto de la población.

Esta mayor incidencia puede ser explicada por un período más largo de comprobación (hasta 20 años) y tal vez por la inclusión de diagnósticos más exhaustivos (por ejemplo, algunas anomalías tegumentarias) en comparación para la mayoría de los estudios.

\section{Opiniones de Expertos}

Robin M Lally ${ }^{48}$ en 2007, escribió una opinión sobre la "Exposición profesional a agentes antineoplásicos: ¿Cuáles son los riesgos sobre la salud reproductiva?”, basándose en publicaciones sobre el tema como la que se observó en un análisis retrospectivo de los datos de la encuesta de 2.976 farmacéuticos y enfermeras, encontrando que las personas que manejan quimioterápicos antes o durante el embarazo, tenían un riesgo estadísticamente significativo de aborto espontáneo en comparación con aquellos que no lo hicieron. Un dato similar también se observó entre mujeres embarazadas de enfermeros o farmacéuticos que manejan quimioterápicos (Valanis et al. ${ }^{34}, 1999$ ).

Una revisión sistemática y un meta-análisis (Dranitsaris et al. ${ }^{13}$, 2005) de estudios realizados entre 1966-2004 también encontró un pequeño aumento del riesgo de aborto involuntario para las enfermeras expuestas a la quimioterapia, pero no un aumento del riesgo de malformaciones congénitas o nacidos muertos.

Sin embargo, un estudio reciente de 1.519 enfermeras que trabajaban en servicios de Oncología entre 1990-1997 y que utilizaban las medidas de protección que dicta la 
OSHA, encontró que la exposición de la piel a la quimioterapia se asoció con un mayor tiempo para quedarse embarazada, parto prematuro y bajo peso al nacer, en comparación a un grupo de referencia de las enfermeras que estaban expuestas a la quimioterapia. Anomalías tales como aborto involuntario y muerte fetal no estaban relacionados con la exposición a agentes quimioterápicos (Fransman et al. ${ }^{37}, 2007$ ).

Wilson Cañón Montañez et $\mathrm{al}^{46}$ en 2010 , llevaron a cabo una revisión narrativa de la literatura. Se hicieron varios estudios para evaluar desenlaces reproductivos y eventos tóxicos relacionados con la exposición a agentes antineoplásicos como el de Dranitsaris et al. ${ }^{13}$, Frasman et al. ${ }^{37} 2007$ y otros.

Tabla 3. Resumen de efectos estudiados producidos por agentes citostáticos

\begin{tabular}{|c|c|c|c|}
\hline EFECTOS ESTUDIADOS & AUTOR & AÑ̃O & TIPO ESTUDIO \\
\hline $\begin{array}{l}\text { - Malformaciones Congénitas. } \\
\text { - Fetos muertos. } \\
\text { - Abortos espontáneos. }\end{array}$ & George Dranitsaris et al ${ }^{13}$. & 2005 & Meta-análisis \\
\hline $\begin{array}{l}\text { - Mayor tiempo para concebir. } \\
\text { - Bajo peso al nacer. }\end{array}$ & Wouter Frasman et $\mathrm{al}^{25}$. & 2007 & Casos y Control \\
\hline - Abortos espontáneos. & Cristina C Lawson et $\mathrm{al}^{49}$. & 2012 & Cohorte \\
\hline $\begin{array}{l}\text { - Cáncer de mama. } \\
\text { - Cáncer de recto. } \\
\text { - Leucemia, otros tipos de cáncer. } \\
\text { - Recién nacidos: mayor riesgo de anomalías } \\
\text { congénitas oculares y musculo-esqueléticas. } \\
\text { - Muerte fetal, bajo peso al nacer, } \\
\text { prematuridad, otras alteraciones congénitas } \\
\text { excepto sífilis. }\end{array}$ & Pamela A Ratner et $\mathrm{al}^{50}$. & 2010 & Cohorte \\
\hline $\begin{array}{l}\text { - Menor número de anomalías congénitas. } \\
\text { - Bajo peso al nacer. }\end{array}$ & Laura T Arbour et $\mathrm{al}^{47}$. & 2010 & Cohorte \\
\hline - Abortos espontáneos. & Robin M Lally ${ }^{48}$. & 2007 & $\begin{array}{l}\text { Opinión de } \\
\text { expertos }\end{array}$ \\
\hline $\begin{array}{l}\text { - Abortos espontáneos. } \\
\text { - Bajo peso al nacer. } \\
\text { - Leucemia Mieloide aguda y Leucemia } \\
\text { Mieloide crónica. }\end{array}$ & Wilson Cañón Montañez ${ }^{46}$. & 2010 & $\begin{array}{l}\text { Revisión narrativa } \\
\text { de la literatura }\end{array}$ \\
\hline
\end{tabular}

\section{DISCUSIÓN/CONCLUSIONES}

Los efectos carcinogénicos, genotóxicos y de toxicidad reproductiva que ejercen los agentes citostáticos sobre profesionales expuestos (enfermería y farmacéuticos sobretodo, y en menor medida personal de lavandería, limpieza y transporte), han quedado establecidos a través de múltiples trabajos. En profesionales, aunque las dosis de exposición son menores que las dosis terapéuticas de pacientes, se ha visto que las vías de absorción pueden ser varias (inhalación, dérmica, enteral y parenteral) y que el daño viene inducido por dosis acumulativas en el tiempo. Dicha exposición queda patente por la detección de metabolitos de las sustancias en cuestión en orina y sangre del personal expuesto. A su vez, se han detectado restos de citostáticos en superficies de viales, guantes, suelos...

La presente revisión se ha centrado en los efectos de toxicidad reproductiva sobre el colectivo de enfermería principalmente. Ha quedado establecido en los diferentes estudios el hecho de que las enfermeras expuestas a citostáticos tardan más en conseguir la concepción. En cuanto a la tasa de abortos espontáneos también parece haber consenso entre los diferentes estudios en su mayor incidencia, estando en un extremo unos autores que duplican su frecuencia a la de nacidos vivos, y en el otro extremo un grupo que concluyen que hay un pequeño riesgo. Han quedado establecidos en uno de los trabajos 
los factores que pueden coadyuvar a los abortos espontáneos, como son la edad, el abuso de cafeína, alcohol y tabaco, y la turnicidad nocturna.

En el tema de malformaciones congénitas hay discrepancias, unos autores apoyan la relación y otros afirman que no hay asociación estadísticamente significativa. Tampoco se ha llegado a establecer una evidencia entre los citostáticos y los embarazos ectópicos, los partos prematuros o el bajo peso al nacer.

Tras realizar la revisión y obtener los anteriores resultados, se abren varias líneas de debate y/o futura investigación:

1. Los resultados encontrados no muestran una asociación estadística significativa causa-efecto, y por lo tanto sería de utilidad realizar estudios más potentes (con más población, en periodos más largos y más dirigidos, o sea descartando otros agentes de exposición como puedan ser gases anestésicos o radiaciones ionizantes).

2. Desde 2004 existen publicados múltiples protocolos y guías de Manejo Seguro de Citostáticos, desde organizaciones internacionales como el NIOSH (National Institute for Occupational Safety and Health: Preventing Occupational Exposure to Antineoplastic and Other Hazardous Drugs in Health Care Settings, 2004), o la OSHA (Occupational Safety and Health Administration: Controlling Occupational Exposure To Hazardous Drugs, 1999), a las nacionales como la guía del Ministerio de Sanidad en el marco del Plan Nacional de Calidad (Prácticas para mejorar la seguridad de los medicamentos de alto riesgo, 2007), la del INSHT (NTP740: Exposición Laboral a citostáticos en el ámbito sanitario, 2007) u otras publicadas por Asociaciones Científicas o de Enfermería (e.g.ANMTAS), Mutuas, la Industria Farmacéutica,... En dichos protocolos se proponen medidas de protección ambiental (diseño de las zonas de trabajo para la preparación, cabinas de flujo laminar, uso de sistemas cerrados para la dispensación, y de materiales de bioseguridad, kits de limpieza ante vertidos accidentales, contenedores específicos para el material desechable, ...) y equipos de protección individual (guantes y mascarillas específicas, bata y gorro). A pesar de la existencia de dichas directrices, los estudios posteriores al meta-análisis de 2005 siguen evidenciando riesgos y efectos relacionados con la exposición, por lo tanto existe un margen para mejorar la evaluación del cumplimiento de dichos protocolos (evaluación de riesgos, formación e información, registro de personal expuesto, diseño de check-lists, ...)

3. En el apartado de Vigilancia de la Salud, se podría mejorar realizando exámenes de salud periódicos, donde se detectara personal especialmente sensible al manejo de citostáticos, y exámenes de salud de nueva incorporación.

4. Aunque se menciona tangencialmente en algún estudio, no hay literatura sobre los efectos de los citostáticos sobre profesionales varones (posible azoospermia, oligospermia, ...) o sobre los cónyuges de los profesionales expuestos, por lo tanto esta podría ser una línea de futura investigación.

\section{BIBLIOGRAFÍA}

1. International Agency for Research on Cancer. (2001): Monographs on the Evaluation of the Carcinogenic Risk of Chemicals to Humans: Pharmaceutical Drugs. IARC, Lyon, France.

2. Barlow SM, Sullivan FM: Reproductive hazards of industrial chemicals. New York, NY: Academic Press, 1982.

3. Hemminki K, Sorsa M, Vainio H: Genetic risks caused by occupational chemicals: use of experimental methods and occupation al risk group monitoring in the detection of environmental chemicals causing mutations, cancer and malformations. Scand J Work Environ Health 1979; 5:307-27.

4. Hatem A. Azim Jr. Fedro A. Peccatori. Nicholas Pavlidis: Treatment of the pregnant mother with cancer: A systematic review on the use of cytotoxic, endocrine, targeted agents and immunotherapy during pregnancy. Part I: Solid tumors. Cancer Treatment Reviews 36 (2010) 101-109. 
5. Hatem A. Azim Jr. Fedro A. Peccatori. Nicholas Pavlidis: Treatment of the pregnant mother with cancer: A systematic review on the use of cytotoxic, endocrine, targeted agents and immunotherapy during pregnancy. Part II. Hematological tumors. Cancer Treatment Reviews 36 (2010) 110-121.

6. Cardonick E, Iacobucci A: Use of chemotherapy during human pregnancy. Lancet Oncol. 2004;5:283-291.

7. Ebert U, Loffler H, Kirch W: Cytotoxic therapy and pregnancy. Pharmacol Ther. 1997;74:207-220.

8. Zemlickis D, Lishner M, Degendorfer P, et al: Fetal outcome after in utero exposure to cancer chemotherapy. Arch Intern Med. 1992;152:573-576.

9. P. V. Rekhadevi, N. Sailaja, M. Chandrasekhar, M. Mahboob, M. F. Rahman and Paramjit Grover: Genotoxicity assessment in oncology nurses handling antineoplastic drugs. Mutagenesis vol. 22 no. 6 pp. 395-401, 2007.

10. James Couch. Derry Stover. Chandran Achutan: Occupational Exposures to Antineoplastic Drugs in an Oncology-Hematology Department. 2011. Journal of Occupational and Environmental Hygiene, 8: D1-D6.

11. National Institute for Occupational Safety and Health. (2004): NIOSH alert: Preventing occupational exposures to antineoplastic and other hazardous drugs in health care settings. [DHHS (NIOSH) Publication No. 2004-165]. Cincinnati, OH: NIOSH Publications Dissemination.

12. Cavallo D, Ursini CL, Perniconi B, Francesco AD, Giglio M, Rubino FM, Marinaccio A, Iavicoli S: Evaluation of genotoxic effects induced by exposure to antineoplastic drugs in lymphocytes and exfoliated buccal cells of oncology nurses and pharmacy employees. Mutat Res 2005, 587:45-51.

13. Dranitsaris G, Johnston M, Poirier S, Schueller T, Milliken D, Green E, Zanke B: Are health care providers who work with cancer drugs at an increased risk for toxic events? A systematic review and meta-analysis of the literature. J Oncol Pharm Pract 2005, 11:69-78.

14. Sessink PJ, Bos RP: Drugs hazardous to healthcare workers. Evaluation of methods for monitoring occupational exposure to cytostatic drugs. Drug Saf 1999, 20:347-359.

15. Ündeğer Ü, Baharan N, Kars A, Güç D: Assessment of DNA damage in nurses handling antineoplastic drugs by the alkaline COMET assay. Mutat Res - Genetic Toxicology and Environmental Mutagenesis 1999, 439:277-285.

16. McDevitt JJ, Lees PS, McDiarmid MA: Exposure of hospital pharmacists and nurses to antineoplastic agents. J Occup Med 1993, 35:57-60.

17. Pyy L, Sorsa M, Hakala E: Ambient monitoring of cyclophosphamide in manufacture and hospitals. American Industrial Hygiene Association journal 1988, 49:314-317.

18. Floridia L, Pietropaolo AM, Tavazzani M, Rubino FM, Colombi A: Measurement of surface contamination from nucleoside analogue antineoplastic drugs by high-performance liquid chromatography in occupational hygiene studies of oncologic hospital departments. Journal of chromatography 1999, 724:325-334.

19. Connor TH, Anderson RW, Sessink PJ, Broadfield L, Power LA: Surface contamination with antineoplastic agents in six cancer treatment centers in Canada and the United States. Am J Health Syst Pharm 1999, 56:1427-1432.

20. Larson RR, Khazaeli MB, Dillon HK: Monitoring method for surface contamination caused by selected antineoplastic agents. Am J Health Syst Pharm 2002, 59:270-277.

21. Connor TH, Sessink PJ, Harrison BR, Pretty JR, Peters BG, Alfaro RM, Bilos A, Beckmann G, Bing MR, Anderson LM, Dechristoforo R: Surface contamination of chemotherapy drug vials and evaluation of new vial cleaning techniques: results of three studies. Am J Health Syst Pharm 2005, 62:475-484.

22. Minoia C, Turci R, Sottani C, Schiavi A, Perbellini L, Angeleri S, Draicchio F, Apostoli P: Application of high performance liquid chromatography/tandem mass spectrometry in the environmental and biological monitoring of health care personnel occupationally exposed to cyclophosphamide and ifosfamide. Rapid Commun Mass Spectrom 1998, 12:1485-1493.

23. Ziegler E, Mason HJ, Baxter PJ: Occupational exposure to cytotoxic drugs in two UK oncology wards. Occupational and environmental medicine 2002, 59:608-612.

24. Sessink PJ, Cerna M, Rossner P, Pastorkova A, Bavarova H, Frankova K, Anzion RB, Bos RP: Urinary cyclophosphamide excretion and chromosomal aberrations in peripheral blood lymphocytes after occupational exposure to antineoplastic agents. Mutation research 1994,309:193-199.

25. Fransman W, Vermeulen R, Kromhout H: Occupational dermal exposure to cyclophosphamide in Dutch hospitals: a pilot study. The Annals of occupational hygiene 2004, 48:237-244.

26. Sorsa M, Anderson D: Monitoring of occupational exposure to cytostatic anticancer agents. Mutation research 1996, 355:253-261.

27. Sessink PJ, Van de Kerkhof MC, Anzion RB, Noordhoek J, Bos RP: Environmental contamination and assessment of exposure to antineoplastic agents by determination of cyclophosphamide in urine of 
exposed pharmacy technicians: is skin absorption an important exposure route? Archives of environmental health 1994, 49:165-169.

28. Ensslin AS, Stoll Y, Pethran A, Pfaller A, Rommelt H, Fruhmann G: Biological monitoring of cyclophosphamide and ifosfamide in urine of hospital personnel occupationally exposed to cytostatic drugs. Occupational and environmental medicine 1994, 51:229-233.

29. Sessink PJ, Boer KA, Scheefhals AP, Anzion RB, Bos RP: Occupational exposure to antineoplastic agents at several departments in a hospital. Environmental contamination and excretion of cyclophosphamide and ifosfamide in urine of exposed workers. International archives of occupational and environmental health 1992, 64:105-112.

30. NTP 740: Exposición laboral a citostáticos en el ámbito sanitario. Instituto Nacional de Seguridad e Higiene en el Trabajo. 2006.

31. Selevan SG, Lindbohm ML, Hornung RW, et al: A study of occupational exposure to antineoplastic drugs and fetal loss in nurses. N Engl J Med. 1985;313:1173-1178.

32. Stücker I, Caillard JF, Collin R, et al.: Risk of spontaneous abortion among nurses handling antineoplastic drugs. Scand J Work Environ Health. 1990;16:102-107.

33. Stücker I, Mandereau L, Hémon D: Relationship between birthweight and occupational exposure to cytostatic drugs during or before pregnancy. Scand J Work Environ Health. 1993;19:148-153.

34. Valanis BG, Vollmer WM, Steele P: Occupational exposure to antineoplastic agents: self-reported miscarriages and stillbirths among nurses and pharmacists. J Occup Environ Med. 1999;41:632-638.

35. Hemminki K, Kyyronen P, Lindbohm ML: Spontaneous abortions and malformations in the offspring of nurses exposed to anaesthetic gases, cytostatic drugs, and other potential hazards in hospitals, based on registered information of outcome. J Epidemiol Community Health. 1985;39:141-147.

36. Skov $\mathrm{T}$, Maarup $\mathrm{B}$, Olsen $\mathrm{JH}$, et al.: Leukaemia and reproductive outcome among nurses handling antineoplastic drugs. Br J Ind Med. 1992;49: 855-861.

37. Fransman W, Roeleveld N, Peelen S, de Kort W, Kromhout H, Heederik D: Nurses with dermal exposure to antineoplastic drugs: reproductive outcomes. Epidemiology 2007, 18:112-119.

38. Valanis B. Vollmer W. [_ahuhn K.GIass A. EíTects: "1 exposure to amincoplastic agents on self-reported infertilily among nurses and phannacisls. J (h-i-u/i Ei'.vimn Med 1997:39:574-580

39. McDonald AD, McDonald JG, Armstrong B, et al.Congenital defcts and work pregnancy. Br J Ind Med $1988 ; 45: 581-588$.

40. Saurel-Cubizolles, MJ, Job-Spira N, Estryn-Behar M. Ectopic pregnancy and occupational exposure to antineoplastic drugs. Lancet 1993:341:1169-71.

41. Mc Abee RR, Gallucci BJ, Chechoway H.Adverse reproductive outcomes and occupational exposures among nurses: an investigation of multiple hazardous exposures. AAOHN J 1993;41:110-19.

42. Gunnarsdottir HK, Aspelund T, Karlsson T, Rafnsson V. Occcupational risk factors for breast cancer among nurses. Int J Occup Environ Health 1997;3:254-258.

43. Bouyer J, Saurel-Cubizolles MJ, Grenier C, et al. Ectopic pregnancy and occupational exposure of hospital personnel. Scand j Work Environ health 1998;24:98-103.

44. Peelen S, Roeveld N, Heederick C., et al. Toxic effects on reproduction in hospital personnel. Dutch ministry of social Affairs and Employment. 1999 (Translation from Dutch to English by Interverbum, Gotheberg, Sweden).

45. Lorente C, Cordier S, Bergeret A, et al. Maternal occupational risk factors for oral clefts. Occupational exposures and Congenital Malformation Working Group. Scand j Work Environ health 2000;26:137-45.

46. Wilsón canon Montañez y Alba Luz Rodriguez-Acelas. ¿Se incrementa el riesgo de cáncer, complicaciones reproductivas y eventos tóxicos en trabajadores de la salud expuestos a agentes antineoplásicos? Revista de Enfermeria Clínica. 2010;20(3):208-210.

47. Laura T Arbour, MD, Kris Beking; MSc, Nhu D.Le, Pamela A Ratner, John J. Spinelli, Kay Teschke, Richard P. Gallagher, MA, Zenaida U. Abanto, Helen Dimich-Ward.Rates of Congenital Anomalies and Other Adverse Birth Outcomes in an Offspring Cohort of Registered Nurses from British Columbia, Canada. Can J Public Health 2010;101 (3):230-234.

48. Robin M Lally. Occupational Exposure to Antineoplastic Agents: What are the reproductive Health Risks? ONS Connect; Oct 2007; 22,10; Proquest Health \& Medical Complete, p. 22.

49. Christina C. Lawson, Carissa M. Rocheleau, Elizabeth A. Whelan, Eileen N. Lividoti Hilbert, MA, Barbara Grajewski, Donna Spiegelman, Janet W.Rich-Edwards.Occupational Exposures among nurses and risk of spontaneous abortion. Am J Obstet Gynecol 2012; 206:327. E 1-8. 
50. Pamela a Ratner, John J Spinelli, Kris Beking, Maria Lorenzi, Chow Yat, Kay Teschke, D Le Nhu, Richard Gallegeher y Helen Dimich-Ward.Cancer Incidence and Adverse Pregnancy Outcome in Registered Nurses Potentially Exposed to Antineoplastic Drugs.BMC Nursing 2010;9:15.

51. Crauste-Manciet, S., Sessink, P.J.M., Ferrari, S., Jomier, J.-Y. Y Brossard, D. (2005). Environmental Contamination with Cytotoxic Drugs in Healthcare Using Positive Aire Pressure Isolators. Ann. occup. Hyg. 49 (7): 619-628.

52. Evelo, C.Ta., Bos, R.P., Peters, J.G.P.Y Henderson P.Th. (1986). Urinary cyclophosphamide assay as a method for biological monitoring of occupational exposure to cyclophosphamide. Int. Arch. Occup. Environ. Health 58:151-155.

53. Favier, B., Gilles, L., Ardiet C. Y Latour, J.F (2003). External contamination of vials containing cytotoxic agents supplied by pharmaceutical manufacturers. J. Oncol. Pharm. Practice 9:15-20.

54. Hedmer, M., Georiadi, A., Rámme-Bremberg, E., Jonsson, B.A.G., Eksborg, S. (2005). Surface contamination of Cyclophosfamide packaging and surtace contamination with antineoplastic drugs in a hospital pharmacy in Sweeden. Ann. Occup. Hyg. 49(7): 629-637.

55. Kiffmeyer, T.K., Kube, C., Opiolka, S., Schmidt, K.G Y Sessink, P.J.M. (2002). Vapour pressures, evaporation behaviour and airborne concentrations of hazardous drugs: implications for occupational safety. The Pharm. J. 268: 331-337.

56. Mason, H.J., Morton, J., Garfitt, S.J., Iqbal, S., Jones, K. (2003). Cytotoxic drug contamination on the outside of vials delivered to a hospital pharmacy, Ann. Occup. Hyg. 47(8): 681-685.

57. Nygren, O., Gustavssson, B., Strom, L., Friberg, A.(2002). Cisplatin contamination observed on the Outside of drug vials. Ann. Occup. Hyg. 46(6): 555-557.

58. Pethran, A., Schierl, R., Hauff, K., Grimm, C.H. Y Boos K.S. (2003). Uptake of antineoplastic agents in pharmacy and hospital personnel. Part I: monitoring of urinary concentrations. Int. Arch. of Occup. Environ. Health 76: 5-10.

59. Rosell Farrás M. G et al. (2006). Antineoplastic Exposure. Assesment and Safety Procedures in Health Care Settings. En Mondelo, P; Mattila, M.; Karwowski, W.; Hale, A. "Proceedings of the Fourth International Conference on Occupational Risk Prevention" (2006) ISBN 84-933328-9-5.

60. Schulz, H., Bigelow, S., Dobish, R., Chambers, C. R. (2005). Antineoplastic agent workplace contamination study: the Alberta Cancer Board Pharmacy perspective. J. Oncol. Pharm. Practice 11: 101-109.

61. Ziegler, E., Mason, H.J. y Baxter, P.J. (2002). Occupational exposure to cytotoxic drugs in two UK oncology wards. Occup. Environ. Med. 59: 608-612.

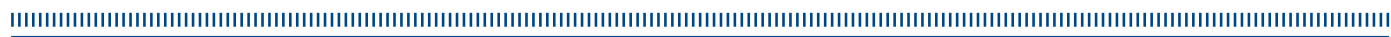




\section{ANEXOS}

Anexo 1. Niveles de citostáticos en suelos y superficies de trabajo

\begin{tabular}{|c|c|c|}
\hline Referencia & Citostático & $\mathrm{ng} / \mathrm{cm}^{2}$ \\
\hline \multirow{4}{*}{51} & Ciclofosfamida & $<0.009-6.6$ \\
\hline & Ifosfamida & $<0.009-0.85$ \\
\hline & 5-Fluorouracilo & $<1.8-88$ \\
\hline & Metotrexato & $<0.9-8.6$ \\
\hline \multirow{4}{*}{61} & Platino & $10-4-0.1$ \\
\hline & Ciclofosfamida & ND - 0.1 \\
\hline & Ifosfamida & ND -0.05 \\
\hline & Metotrexato & $11-19$ (superficie total) \\
\hline 55 & Ciclofosfamida & ND - 190 \\
\hline 29 & 5-Fluorouracilo & $<0.1-11$ \\
\hline 59 & Ciclofosfamida & 0.01 \\
\hline
\end{tabular}

Anexo 2. Niveles de citostáticos en guantes (por par)

\begin{tabular}{cll}
\hline Referencia & Citostático & Cantidad \\
\hline \multirow{2}{*}{51} & Ciclofosfamida & $<0.009 \mathrm{nq}-9 \mathrm{ug}$ \\
& Ifosfamida & $<0.009 \mathrm{ng}-930 \mathrm{ug}$ \\
& 5-Fluorouracilo & $<1.8 \mathrm{ng}-359 \mathrm{ug}$ \\
& Metotrexato & $<0.9 \mathrm{ng}-1.6 \mathrm{ug}$ \\
\cline { 2 - 3 } & Platino & $<1 \mathrm{ng}-36 \mathrm{ug}$ \\
$\mathbf{6 1}$ & Ciclofosfamida & ND -11.2 \\
& Ifosfamida & ND $-1.8 \mathrm{ug}$ \\
& Metotrexato & ND $-49.3 \mathrm{ug}$ \\
\cline { 2 - 3 } & Ciclofosfamida & $<80 \mathrm{ng}-9.6 \mathrm{ug}$ \\
& 5-Fluorouracilo & $<4 \mathrm{mg}-760 \mathrm{ug}$ \\
& Metotrexato & $<11 \mathrm{mg}-1.9 \mathrm{mg}$ \\
\cline { 2 - 2 } & Ciclofosfamida & $<0.1 \mathrm{mg}-21 \mathrm{ug}$ \\
& 5-Fluorouracilo & $19 \mathrm{mg}-87 \mathrm{ug}$ \\
& Metotrexato & $<6 \mathrm{mg}-49 \mathrm{ug}$ \\
\hline
\end{tabular}

Anexo 3. Niveles en superficies de viales

\begin{tabular}{|c|c|c|c|}
\hline Referencia & Tapones & Citostático & $\mathrm{ng} / \mathrm{cm}^{2}$ \\
\hline \multirow{2}{*}{57} & \multirow[t]{2}{*}{ Viales } & Cisplatino & $0.2-99$ (en total) \\
\hline & & Cisplatino & $0.6-21$ (en total) \\
\hline \multirow{7}{*}{53} & \multirow{5}{*}{ Viales } & Etopósido & $2.9-18.5$ \\
\hline & & 5-Fluorouracilo & $2.5-15.3$ \\
\hline & & Ifosfamida & 0.1 \\
\hline & & Ciclofosfamida & $<0.1-0.1$ \\
\hline & & Doxorubicina & $<0.1-0.2$ \\
\hline & \multirow{2}{*}{ Envase Exterior } & 5-Fluorouracilo & 0.5 \\
\hline & & Etopósido & ND \\
\hline \multirow{5}{*}{56} & & Carboplatino & $7-251$ (en total) \\
\hline & & Cisplatino & ND - 9 (en total) \\
\hline & & Ciclofosfamida & ND - 39 (en total) \\
\hline & & Ifosfamida & ND - 344 (en total) \\
\hline & & Metotrexato & ND - 18(en total) \\
\hline
\end{tabular}




\begin{tabular}{|c|c|c|c|}
\hline Referencia & Tapones & Citostático & $\mathrm{ng} / \mathrm{cm}^{2}$ \\
\hline \multirow{2}{*}{54} & Envase Exterior & $\begin{array}{l}\text { Ciclofosfamida } \\
\text { Ifosfamida }\end{array}$ & $\begin{array}{l}0.5-3.2 \text { (en total) } \\
\text { ND }-10 \text { (en total) }\end{array}$ \\
\hline & Viales & $\begin{array}{l}\text { Ciclofosfamida } \\
\text { Ifosfamida }\end{array}$ & $\begin{array}{l}13-19 \text { (en total) } \\
1.6-24 \text { (en total) }\end{array}$ \\
\hline \multirow[t]{2}{*}{60} & Viales & Ciclofosfamida & $0.5-2.89$ \\
\hline & $\begin{array}{l}\text { Viales } \\
\text { Tapones }\end{array}$ & $\begin{array}{l}\text { Ciclofosfamida } \\
\text { Ciclofosfamida }\end{array}$ & $\begin{array}{l}0.004-0.1 \\
<0.001-0.5\end{array}$ \\
\hline 59 & Envase Exterior & Ciclofosfamida & $<0.001$ \\
\hline
\end{tabular}

Anexo 4. Niveles de Citostáticos detectados en orina

\begin{tabular}{cll}
\hline Referencia & Citostático & Orina \\
\hline $\mathbf{5 2}$ & Ciclofosfamida & ND $-2.5 \mathrm{mg} / 24 \mathrm{~h}$ \\
\cline { 2 - 3 } 29 & 5-Fluorouracilo & ND $-0.5 \mathrm{mg} / 12 \mathrm{~h}$ \\
\cline { 2 - 3 } 27 & Ciclofosfamida & ND $-19.4 \mathrm{mg} / 24 \mathrm{~h}$ \\
\cline { 2 - 3 } & Ciclofosfamida & ND $-38.23 \mathrm{mg} / 24 \mathrm{~h}$ \\
& Ifosfamida & ND $-12.74 \mathrm{mg} / 24 \mathrm{~h}$ \\
\cline { 2 - 3 } & Ciclofosfamida & ND $-0.76 \mathrm{mg} / \mathrm{l}$ \\
& Ifosfamida & ND $-1.90 \mathrm{mg} / 1$ \\
& Doxorubicina & ND $-127 \mathrm{ng} / \mathrm{I}$ \\
& Epirubicina & ND $-182 \mathrm{ng} / \mathrm{l}$ \\
\hline
\end{tabular}

